\section{Treatment Stage Migration Maximizes Survival Outcomes in Patients with Hepatocellular Carcinoma Treated with Sorafenib: An Observational Study}

\author{
Clarence Yen $^{a} \quad$ Rohini Sharma $^{a}$ Lorenza Rimassa $^{b} \quad$ Tadaaki Arizumic $^{c}$ \\ Dominik Bettinger ${ }^{d}$ e Huay Yee Choo ${ }^{a}$ Tiziana Pressiani ${ }^{b}$ \\ Michela E. Burlone ${ }^{f}$ Mario Pirisi $^{f}$ Laura Giordano $^{b}$ Anisa Abdulrahman ${ }^{a}$ \\ Masatoshi Kudo ${ }^{c}$ Robert Thimmed, e Joong Won Park ${ }^{g}$ \\ David James Pinato ${ }^{a}$ \\ a Department of Surgery and Cancer, Imperial College London, Hammersmith Hospital, \\ London, UK; ${ }^{b}$ Medical Oncology and Haematology Unit, Humanitas Cancer Center, \\ Humanitas Clinical and Research Center, Milan, Italy; ' $D$ Department of Gastroenterology \\ and Hepatology, Kindai University School of Medicine, Osakasayama, Japan; ${ }^{d}$ Department \\ of Medicine II, University Medical Center, and e Berta Ottenstein Programme, Faculty of \\ Medicine, University of Freiburg, Freiburg, Germany; ${ }^{f}$ Department of Translational Medicine, \\ Università degli Studi del Piemonte Orientale "A. Avogadro," Novara, Italy; ${ }^{9}$ Center for Liver \\ Cancer, National Cancer Center Hospital, Goyang, South Korea
}

\section{Keywords}

Hepatocellular carcinoma · Sorafenib - Treatment stage migration · Prognosis ·

Treatment-naïve patients

\begin{abstract}
Background: Level I evidence supports the use of sorafenib in patients with Barcelona Clinic Liver Cancer (BCLC) stage $C$ hepatocellular carcinoma, where heterogeneity in efficacy exists due to varying clinicopathologic features of the disease. Aim: We evaluated whether prior treatment with curative or locoregional therapies influences sorafenib-specific survival. Methods: From a prospective data set of 785 consecutive patients from international specialist centres, 264 patients (34\%) were treatment naïve (TN) and 521 (66\%) were pre-treated (PT), most frequently with transarterial chemoembolization ( $n=413 ; 79 \%)$. The primary endpoint was overall survival (OS) from sorafenib initiation with prognostic factors tested on uni- and multivariate analyses. Results: Median OS for the entire cohort was 9 months; the median sorafenib duration was 2.8 months, with discontinuation being secondary to progression $(n=$
\end{abstract}

Dr. David James Pinato, MD, MRes, MRCP (UK), PhD 
454; $58 \%$ ) or toxicity ( $n=149 ; 19 \%$ ). PT patients had significantly longer OS than TN patients (10.5 vs. 6.6 months; $p<0.001$ ). Compared to TN patients, PT patients had a better Child-Pugh $(C P)$ class (CP A: 57 vs. 47\%; $p<0.001$ ) and a lower BCLC stage (BCLC A-B, 40 vs. $30 \% ; p=$ $0.007)$. PT status preserved an independent prognostic role $(p=0.002)$ following adjustment for BCLC stage, $\alpha$-fetoprotein, CP class, aetiology, and post-sorafenib treatment status. PT patients were more likely to receive further anticancer treatment after sorafenib (31 vs. $9 \%$; $p<$ 0.001). Conclusion: Patients receiving sorafenib after having failed curative or locoregional therapies survive longer and are more likely to receive further treatment after sorafenib. This suggests an incremental benefit to OS from sequential exposure to multiple lines of therapy, justifying treatment stage migration in eligible patients.

(c) 2017 S. Karger AG, Basel

\section{Introduction}

Hepatocellular carcinoma (HCC) is the third most common cause of cancer-related death worldwide at a mortality-to-incidence ratio of $0.95[1,2]$ and 5-year survival rates ranging from 5 to 20\% [3]. Treatment options for HCC have widened and improved over time to include surgical, locoregional, and systemic therapies [4], and staging algorithms including the Barcelona Clinical Liver Cancer (BCLC) system have facilitated a rational treatment allocation process. Despite increasing efforts addressed at harmonizing management decisions, there is recognized geographical variation in the provision of radical and palliative treatments in HCC where regional preferences and the availability of each treatment modality among the treating multidisciplinary tumour board affect outcomes $[5,6]$.

The last decade has seen sorafenib emerging as the first systemic agent to produce a survival benefit of approximately 3 months in the treatment of advanced HCC $[7,8]$. The magnitude of the survival benefit documented in pre-registration trials is extrapolated from a population of patients with advanced HCC, Child-Pugh (CP) class A, for whom sorafenib represented the first-line option for systemic therapy [9].

In clinical practice, the provision of sorafenib is not solely restricted to treatment-naïve (TN) patients who present with advanced HCC and preserved liver function, but also extends to patients who have progressed after curative or locoregional therapies. The provision of sorafenib as the next most suitable therapy in the context of disease progression or patient ineligibility to further locoregional therapies, a concept also termed "treatment stage migration" [10], is globally the most common indication for the use of sorafenib, as shown in the GIDEON study, where $57 \%$ of the patients on sorafenib had previously received local surgical ablative therapy or transarterial chemoembolization (TACE) [11]. Subanalyses of pre-registration trials have initially demonstrated that sorafenib treatment leads to a significant survival advantage over placebo in patients with HCC irrespective of a number of key clinicopathologic features, including disease aetiology, baseline tumour burden, performance status, tumour stage, and prior therapy $[12,13]$. However, it is unclear whether TN patients have a life expectancy similar to that of patients who are migrated to sorafenib after failure of radical/locoregional therapies in routine clinical care, where provision of sorafenib does not strictly follow clinical trial eligibility criteria and often extends to subjects with a wider range of liver functional reserve and BCLC stage, variables that are likely to make the expected survival benefit from sorafenib dissimilar to that reported in phase III trial data $[14,15]$. Moreover, since the treatment landscape of HCC has recently expanded to include second-line therapies $[16,17]$, it is important to understand whether treatment sequencing prior to sorafenib might influence the eligibility of patients to receive further systemic treatment lines following sorafenib discontinuation [18]. 
Yen et al.: Treatment Stage Migration Maximizes Survival Outcomes in Patients with Hepatocellular Carcinoma Treated with Sorafenib: An Observational Study

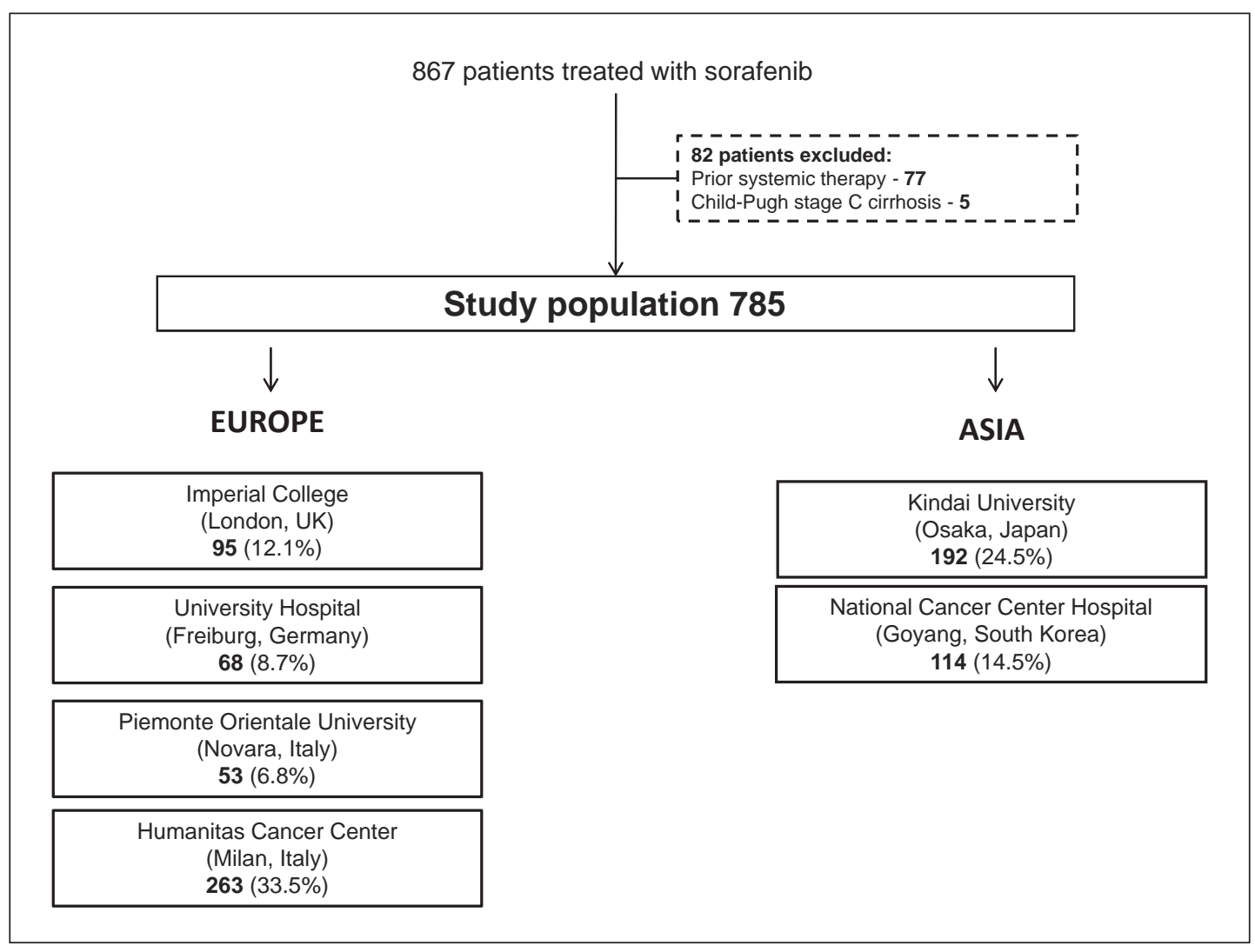

Fig. 1. Study flow chart.

To address these issues, we designed this study aiming to compare and contrast the clinicopathologic features and survival outcomes of patients who received sorafenib for two different clinical indications: (1) as first-line therapy for previously untreated HCC (TN group) or (2) in the context of disease progression or relapse following prior treatment with radical or locoregional therapies (PT group).

\section{Methods}

This observational study was conducted on a prospectively maintained, multicentre data set of 867 consecutive patients receiving sorafenib from 6 tertiary referral centres with HCC multidisciplinary services between 2008 and 2016. From this database, 77 patients who had previously received systemic therapy and 5 CP class $\mathrm{C}$ patients were excluded.

The final data set of 785 patients consisted of 449 patients (61.0\%) from Europe and 306 (39.0\%) from Asia. The European centres included 95 patients (12.1\%) from Imperial College London (UK), 68 patients (8.7\%) from University Hospital Freiburg (Germany), 53 patients (6.8\%) from the Academic Liver Unit in Novara (Italy), and 263 patients (33.5\%) from the Humanitas Cancer Center in Milan (Italy). The Asian subgroup included 192 patients (24.5\%) from Kindai University, Osakasayama (Japan), and 114 patients (14.5\%) from the National Cancer Center Hospital, Goyang (South Korea) (Fig. 1). All patients met the criteria for a histological and/or radiological diagnosis of HCC [10].

Sorafenib was administered after multidisciplinary discussion, either as the first anticancer therapy or in the setting of relapse, failure, or ineligibility to radical or locoregional treatments. The clinical follow-up of the patients during sorafenib treatment included routine blood tests, physical examination, and adverse event assessment before each cycle of sorafenib. Radiologic staging was performed using computerized 


\section{Liver Cancer}

Table 1. Clinicopathologic features at study baseline

\begin{tabular}{l|l}
\hline Liver Cancer 2017;6:313-324 \\
\hline DOI: 10.1159/000480441 & $\begin{array}{l}\text { ( 2017 S. Karger AG, Basel } \\
\text { www.karger.com/lic }\end{array}$ \\
\hline
\end{tabular}

Yen et al.: Treatment Stage Migration Maximizes Survival Outcomes in Patients with Hepatocellular Carcinoma Treated with Sorafenib: An Observational Study

\begin{tabular}{|c|c|c|c|}
\hline Patient characteristic & $\begin{array}{l}\text { Treatment naïve } \\
n=264\end{array}$ & $\begin{array}{l}\text { Prior treatment } \\
n=521\end{array}$ & $p$ value \\
\hline $\begin{array}{l}\text { Median age at sorafenib } \\
\text { initiation (range), years }\end{array}$ & $68.6(59.9-76.2)$ & $69.5(61.4-76.6)$ & 0.309 \\
\hline \multicolumn{4}{|l|}{ Gender } \\
\hline Male & $204(77.3 \%)$ & $426(81.8 \%)$ & 0.135 \\
\hline Female & $60(22.7 \%)$ & $95(18.2 \%)$ & \\
\hline \multicolumn{4}{|l|}{ Aetiology } \\
\hline Hepatitis B virus & $58(22.0 \%)$ & $125(24.0 \%)$ & 0.003 \\
\hline Hepatitis $\mathrm{C}$ virus & $76(28.8 \%)$ & $203(39.0 \%)$ & \\
\hline Non-viral & $130(49.2 \%)$ & $193(37.0 \%)$ & \\
\hline \multicolumn{4}{|l|}{ Geography } \\
\hline Western & 197 (74.6\%) & $282(54.1 \%)$ & $<0.001$ \\
\hline Eastern & $67(25.4 \%)$ & $239(45.9 \%)$ & \\
\hline \multicolumn{4}{|l|}{ Extrahepatic spread } \\
\hline Absent & $162(63.3 \%)$ & 317 (61.4\%) & 0.619 \\
\hline Present & $94(36.7 \%)$ & 199 (38.6\%) & \\
\hline \multicolumn{4}{|l|}{ Portal vein invasion } \\
\hline Absent & $170(64.4 \%)$ & 397 (76.2\%) & $<0.001$ \\
\hline Present & $94(35.6 \%)$ & $124(23.8 \%)$ & \\
\hline \multicolumn{4}{|l|}{$\alpha$-Fetoprotein } \\
\hline$>400 \mathrm{ng} / \mathrm{mL}$ & $121(46.9 \%)$ & $181(36.0 \%)$ & 0.004 \\
\hline$<400 \mathrm{ng} / \mathrm{mL}$ & 137 (53.1\%) & $322(64.0 \%)$ & \\
\hline \multicolumn{4}{|l|}{ Child-Pugh class } \\
\hline $\mathrm{A}$ & $117(47.2 \%)$ & $283(57.3 \%)$ & 0.009 \\
\hline B & $131(52.8 \%)$ & $211(42.7 \%)$ & \\
\hline \multicolumn{4}{|l|}{ BCLC stage } \\
\hline $\mathrm{A} / \mathrm{B}$ & $80(30.3 \%)$ & $209(40.1 \%)$ & 0.007 \\
\hline $\mathrm{C}$ & $184(69.7 \%)$ & 312 (59.9\%) & \\
\hline $\begin{array}{l}\text { Median duration of } \\
\text { sorafenib treatment } \\
\text { (range), months }\end{array}$ & $2.9(1.6-6.9)$ & $2.6(1.4-6.9)$ & 0.755 \\
\hline \multicolumn{4}{|l|}{ Previous treatment } \\
\hline Liver resection & - & $148(28.4 \%)$ & - \\
\hline Liver transplant & - & $7(1.3 \%)$ & \\
\hline RFA & - & $168(32.2 \%)$ & \\
\hline TACE & - & $413(79.3 \%)$ & \\
\hline
\end{tabular}

Bold type denotes significance. BCLC, Barcelona Clinic Liver Cancer; RFA, radiofrequency ablation; TACE, transarterial chemoembolization.

tomography and/or magnetic resonance imaging, as clinically indicated. The patients were re-assessed radiologically for disease response status every 8-12 weeks using the modified RECIST (mRECIST) criteria on contrast-enhanced imaging.

The patients' clinicopathological characteristics, including treatments received prior to and after sorafenib, were collected, and the baseline CP class and BCLC stage were reconstructed $[19,20]$. The primary endpoint of this study was overall survival (OS), calculated from the date of sorafenib commencement until death or last follow-up. In addition, we performed a subgroup analysis on post-sorafenib treatment status to explore its confounding effect on survival in the PT and TN groups.

The study was performed following research ethics committee approval from all participating institutions in accordance with the good clinical practice standards published in the Declaration of Helsinki. 
Yen et al.: Treatment Stage Migration Maximizes Survival Outcomes in Patients with Hepatocellular Carcinoma Treated with Sorafenib: An Observational Study

Statistical Analysis

The patients' characteristics were analysed by descriptive statistical methods and are presented as means or medians, as appropriate. Normality was tested with the Shapiro-Wilk test. The Pearson $\chi^{2}$ test or Fisher exact test was used for analysis of proportions, as appropriate. Kaplan-Meier curves with log-rank testing were used to perform a univariate survival analysis, with significant factors $(p<0.05)$ being entered into Cox regression models. All statistical analyses were performed using SPSS version 21.0 (IBM Inc., Chicago, IL, USA) and conducted at 95\% confidence intervals (95\% CI), with a two-tailed level of significance at $p<0.05$.

\section{Results}

\section{Patient Characteristics}

A total of 785 patients receiving sorafenib for HCC were included: 521 patients (66.4\%) had been pre-treated with either potentially curative or locoregional treatment (PT group) and 264 patients $(33.6 \%)$ were treatment naïve (TN group). The treatment modalities in the PT group included TACE $(n=413 ; 79.3 \%)$, radiofrequency ablation (RFA; $n=168 ; 32.2 \%)$, and liver resection $(n=148 ; 28.4 \%)$. The mean number of prior treatment lines was 2 , with 282 patients $(54.1 \%)$ having received only 1 prior line of treatment.

The differential distribution of the clinical characteristics of the TN and PT patients is presented in Table 1. There were no differences in age at sorafenib initiation, gender distribution, and extrahepatic spread between the TN and PT cohorts at baseline. The treatment duration was similar across the groups: 2.9 months (95\% CI: 1.6-6.9) in the TN group and 2.6 months (95\% CI: 1.4-6.9) in the PT group ( $p=0.75$ ). Sorafenib discontinuation primarily followed progression of disease $(n=454 ; 58.1 \%)$ and unacceptable toxicity $(n=149 ; 19.0 \%)$. Hepatitis C virus (HCV) aetiology was more prevalent in PT than in TN patients $(n=203$, $39.0 \%$, vs. $n=76,28.8 \% ; p=0.003)$. There were more patients treated in Western centres in the TN group $(n=197 ; 74.6 \%)$ than in the PT group $(n=282 ; 54.1 \%)(p<0.001)$. At baseline, 312 patients $(59.9 \%)$ in the PT group satisfied BCLC stage C criteria, as compared to 184 patients $(69.7 \%)$ in the TN group ( $p=0.007)$. The PT group had a higher proportion of CP class A patients $(n=283 ; 57.3 \%)$ than the TN group $(n=117 ; 47.2 \%)(p=0.009)$, as well as lower $\alpha$-fetoprotein (AFP) levels, with 181 patients (36.0\%) having an AFP level $>400 \mathrm{ng} / \mathrm{mL}$ in the PT group as compared to 121 patients (46.9\%; $p=0.004)$ in the TN group. There were no differences in dose reductions between the two groups $(n=99,38.2 \%$, in the TN group and $n=170,33.0 \%$, in the PT group; $p=0.151$ ).

\section{Prognostic Relationship between Prior Treatment Status and Sorafenib-Specific Survival} in Patients with HCC

Overall, 637 patients (81.1\%) had died by the time of analysis, $233(88.3 \%)$ in the TN group and $404(77.5 \%)$ in the PT group. Median OS across the whole study population was 9.0 months (95\% CI: 8.2-9.7). The patients in the Eastern centres had a median OS of 8.5 months ( $n=306$; 95\% CI: 7.1-9.9 months), while the patients in the Western centres had a non-dissimilar median OS of 9.9 ( $n=479$; 95\% CI: 8.2-10.0 months: $\log$-rank $p=0.074)$. On univariate analysis, the patients in the PT cohort had a significantly longer median OS of 10.5 months (95\% CI: 9.2-11.8), compared to 6.6 months (95\% CI: 5.6-7.6; log-rank $p<0.001)$ in the TN group (Fig. 2a). Other univariate predictors of poorer OS included aetiology of chronic liver disease $(p=0.006)$, AFP $>400 \mathrm{ng} / \mathrm{mL}(p<0.001)$, portal vein invasion (PVI; $p<0.001)$, extrahepatic spread $(p<0.001)$, CP class $(p=0.016)$, and BCLC stage $(p<0.001)$, as shown in Table 2 and Figure 2. The multivariate analyses revealed prior treatment status to remain an independent predictor of OS (HR 1.32; 95\% CI: 1.10-1.57; $p=0.002$ ) following adjustment 


\section{Liver \\ Cancer}

Fig. 2. Kaplan-Meier curves describing the overall survival of patients with hepatocellular carcinoma treated with sorafenib according to prior treatment status (pre-treated or treatment naïve) (a), BCLC stage (b), and aetiology of the underlying cirrhosis (c). BCLC, Barcelona Clinic Liver Cancer; HBV, hepatitis B virus; HCV, hepatitis $C$ virus.

\begin{tabular}{l|l}
\hline \multicolumn{2}{l|}{ Liver Cancer 2017;6:313-324 } \\
\hline DOI: 10.1159/000480441 & $\begin{array}{l}\text { ○ 2017 S. Karger AG, Basel } \\
\text { www.karger.com/lic }\end{array}$ \\
\hline
\end{tabular}

Yen et al.: Treatment Stage Migration Maximizes Survival Outcomes in Patients with Hepatocellular Carcinoma Treated with Sorafenib: An Observational Study

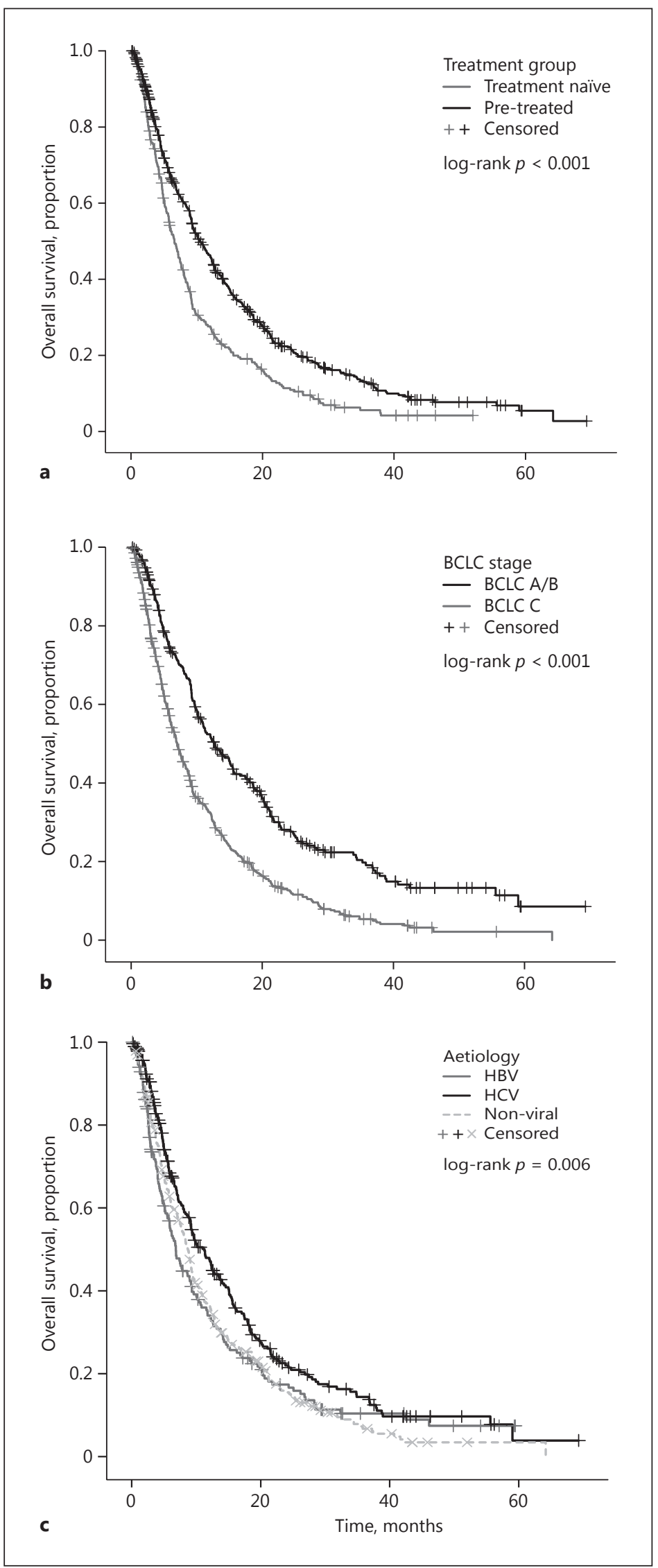


Yen et al.: Treatment Stage Migration Maximizes Survival Outcomes in Patients with Hepatocellular Carcinoma Treated with Sorafenib: An Observational Study

Table 2. Univariate and multivariate analyses of survival

\begin{tabular}{|c|c|c|c|c|c|c|c|}
\hline & $n$ & Univariate & & & Multi & riate & \\
\hline & & OS, months & $95 \% \mathrm{CI}$ & $p$ value & HR & $95 \% \mathrm{CI}$ & $p$ value \\
\hline Gender & & & & & & & \\
\hline Male & 630 & 9.0 & $8.1-9.8$ & 0.292 & - & - & - \\
\hline Female & 155 & 9.2 & $7.2-11.2$ & & & & \\
\hline Aetiology & & & & & & & \\
\hline Non-viral & 323 & 8.7 & $7.8-9.6$ & 0.006 & - & - & 0.103 \\
\hline Hepatitis B virus & 183 & 6.8 & $5.4-8.2$ & & 1.04 & $0.83-1.29$ & \\
\hline Hepatitis $\mathrm{C}$ virus & 279 & 11.3 & $9.4-13.2$ & & 0.84 & $0.69-1.01$ & \\
\hline Geography & & & & 0.074 & - & - & - \\
\hline Western & 479 & 9.1 & $8.2-10.0$ & & & & \\
\hline Eastern & 306 & 8.5 & $7.1-9.9$ & & & & \\
\hline$\alpha$-Fetoprotein & & & & & & & \\
\hline$>400 \mathrm{ng} / \mathrm{mL}$ & 459 & 11.8 & $10.2-13.3$ & $<0.001$ & - & - & $<0.001$ \\
\hline$<400 \mathrm{ng} / \mathrm{mL}$ & 302 & 5.6 & $4.7-6.6$ & & 1.82 & $1.53-2.16$ & \\
\hline Portal vein invasion & & & & & & & \\
\hline Absent & 567 & 9.9 & $8.8-11.1$ & $<0.001$ & - & - & - \\
\hline Present & 218 & 5.9 & $4.7-7.0$ & & & & \\
\hline Extrahepatic spread & & & & & & & \\
\hline Absent & 479 & 9.7 & $8.6-10.8$ & $<0.001$ & - & - & - \\
\hline Present & 293 & 7.0 & $5.7-8.3$ & & & & \\
\hline Child-Pugh class & & & & & & & \\
\hline A & 400 & 9.2 & $7.9-10.5$ & 0.016 & - & - & 0.046 \\
\hline B & 342 & 8.6 & $7.6-9.6$ & & 1.19 & $1.00-1.41$ & \\
\hline BCLC stage & & & & & & & \\
\hline $\mathrm{A} / \mathrm{B}$ & 289 & 12.7 & $10.3-15.2$ & $<0.001$ & - & - & $<0.001$ \\
\hline $\mathrm{C}$ & 496 & 7.0 & $6.1-7.9$ & & 1.65 & $1.38-1.98$ & \\
\hline Treatment group & & & & & & & \\
\hline Pre-treated & 521 & 10.5 & $9.2-11.8$ & $<0.001$ & - & - & 0.002 \\
\hline Treatment naïve & 264 & 6.6 & $5.6-7.6$ & & 1.32 & $1.10-1.57$ & \\
\hline
\end{tabular}

Bold type denotes significance. OS, overall survival; BCLC, Barcelona Clinic Liver Cancer.

for aetiology, CP class, BCLC stage, and AFP level (Table 2). When stratified according to BCLC stage, the prior treatment status was able to identify patient subsets with clinically meaningful differences in survival, ranging from 18.6 months for PT BCLC stage A/B patients to 8 months for TN BCLC stage A/B patients who were unfit to receive radical or locoregional therapies (online suppl. Table 1; see www.karger.com/doi/10.1159/000480441 for all online suppl. material).

We evaluated the relationship between prior treatment status and best type of radiologic response according to the mRECIST criteria. Radiologically proven disease progression was documented in $58 \mathrm{TN}$ patients (63.7\%) and $162 \mathrm{PT}$ patients (59.3\%), with no difference in response rate between the groups $(p=0.22)$ (Table 3$)$.

In the analysis of treatment after sorafenib discontinuation, we excluded patients on ongoing sorafenib $(n=23)$ or in disease remission $(n=7)$. In the entire study cohort, provision of further anticancer treatment $(n=119 ; 24.2 \%)$ was associated with an improved median OS of 18.3 months (95\% CI: 13.8-22.9), as compared to 5.8 months (95\% CI: 5.0-6.6) among 
Yen et al.: Treatment Stage Migration Maximizes Survival Outcomes in Patients with Hepatocellular Carcinoma Treated with Sorafenib: An Observational Study

Table 3. Comparison of radiologic responses and the post-sorafenib treatment status between the pre-treated and treatment-naïve patients

\begin{tabular}{lccc}
\hline & Treatment naïve & Pre-treated & $p$ value \\
\hline Radiologic response & $n=91$ & $n=273$ & \\
Progressive disease & $58(63.7 \%)$ & $162(59.3 \%)$ & 0.220 \\
Stable disease & $28(30.8 \%)$ & $75(27.5 \%)$ & \\
Partial response & $5(5.5 \%)$ & $32(11.7 \%)$ & \\
Complete response & $0(0 \%)$ & $4(1.5 \%)$ & \\
Treatment after sorafenib discontinuation & $n=157$ & $n=365$ & $\mathbf{0 . 0 0 1}$ \\
Best supportive care & $136(90.7 \%)$ & $237(69.3 \%)$ & $\mathbf{0 . 0 5}$ \\
Further anticancer treatment & $14(9.3 \%)$ & $105(30.7 \%)$ & \\
\hline
\end{tabular}

Bold type denotes significance.

the patients who received best supportive care (BSC; $n=373 ; 75.8 \% ; p<0.001$ ), with an HR of 1.8 (95\% CI: 1.4-2.2). In the patient cohort receiving further anticancer therapy, 64 patients (53.8\%) received further systemic therapy, while 76 (63.9\%) of them received locoregional therapy (hepatic arterial infusion chemotherapy or radioembolization), with 21 (17.6\%) receiving both locoregional and systemic therapy after sorafenib. Upon sorafenib discontinuation, the patients in the PT group were more likely to receive further anticancer treatment $(n=105 ; 30.7 \%)$ than the TN patients $(n=14 ; 9.3 \% ; p<0.001$; Table 3$)$.

Lastly, we address the potential confounding effect of post-sorafenib therapy status on OS. Among the patients who received BSC after sorafenib discontinuation, the PT group $(n=$ $237 ; 63.5 \%$ ) had a longer median OS of 7.0 months (95\% CI: $5.2-8.9$ ), as compared to the TN group ( $n=136 ; 36.5 \%)$ with a median OS of 4.9 months (95\% CI: 4.2-5.6; $p=0.007$ ), with an HR of 1.4 (95\% CI: 1.1-1.7) (Fig. 3a). Similarly, among the patients who received further anticancer treatment after sorafenib, the PT group ( $n=105 ; 88.2 \%)$ had a longer median OS of 18.6 months (95\% CI: 16.5-20.8), as compared to 7.0 months (95\% CI: 6.5-7.6) in the TN group ( $n=14 ; 11.8 \% ; p=0.004$; Fig. 3b), with an HR of 2.3 (95\% CI: $1.3-4.2)$. This confirmed that the prognostic stratification imparted by pre-sorafenib treatment status was maintained independently from post-sorafenib treatment.

\section{Discussion}

The multi-targeted tyrosine kinase inhibitor sorafenib has remained the only evidencebased systemic treatment option for patients with HCC for a decade, having been the first compound to demonstrate a significant survival benefit over placebo in two landmark phase III studies $[7,8]$. Whilst the positioning of this treatment has initially coincided with the BCLC stage $C$ category of patients (i.e., patients with metastases or PVI, a performance status of $0-2$, and preserved liver function), the administration of sorafenib has progressively widened to include patients with earlier-stage disease who have progressed or are deemed ineligible to further radical or locoregional therapies.

These indications, defined within the BCLC guidelines as "treatment stage migration" [10], are based on subgroup analyses of clinical trials showing that sorafenib is more efficacious than placebo in advanced HCC irrespective of prior treatment $[12,13]$. In the postsorafenib era, however, inconclusive evidence exists to demonstrate whether PT and TN patient subpopulations might have a different life expectancy whilst on sorafenib treatment 


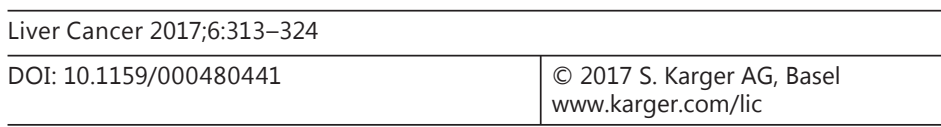

Yen et al.: Treatment Stage Migration Maximizes Survival Outcomes in Patients with Hepatocellular Carcinoma Treated with Sorafenib: An Observational Study

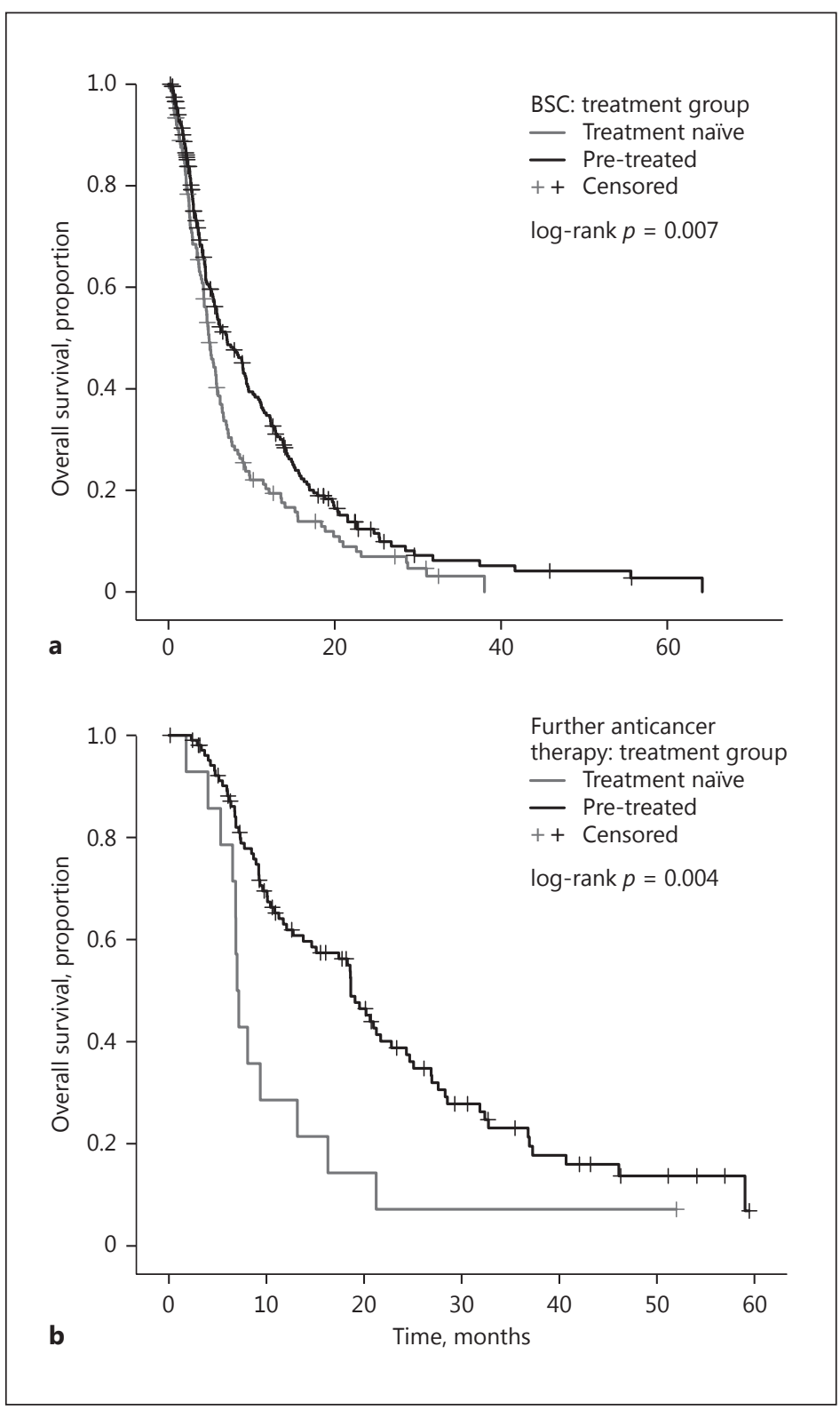

Fig. 3. Kaplan-Meier curves describing the differences in overall survival of patients classified according to prior treatment status following stratification by the type of therapy received after permanent sorafenib cessation: best supportive care (BSC) (a) or further active anticancer therapies (b). 
6 tertiary referral centres across Europe and Asia, we confirmed that sorafenib-specific OS is significantly influenced by the previous anticancer treatment status.

In our study, the patients in the PT group had better liver functional reserve, lower tumour stages, higher AFP levels, and a lower prevalence of PVI, suggesting that the difference in survival observed between the pre-treatment and the treatment-naïve group may be attributed to differences in common clinicopathologic features of the disease [12, 20, 29-33]. However, when we performed multivariate analyses of survival, we found that the 4-month increase in the probability of survival associated with prior treatment was independent of liver functional reserve, stage, aetiology, and AFP levels, which is to suggest that the imbalance of prognostic factors may not entirely explain the difference in survival we observed between the groups.

Our results suggest that patients considered for sorafenib in the context of relapsed/ progressive HCC after radical/locoregional therapies are clinically diverse from those who present with de novo metastatic disease - who, in our study, had shorter OS times despite an equal duration of sorafenib treatment and comparable radiologic responses. We cannot discount the possibility that biologic factors intrinsic to the molecular makeup of HCC might be at the basis of the different survival periods observed between the PT and the TN group, more so in light of recent evidence suggesting genomic diversity in the evolution of HCC [34]. It is possible that in the PT group, the HCC might have had a more indolent course that allowed early detection and facilitated the provision of multiple lines of treatment, leading to better patient selection. On the other hand, the diverse distribution of aetiologic factors across groups might be an equally important factor to underpin such biologic heterogeneity. Interestingly, in our study, we found the PT group to comprise more HCV-related cirrhotics, whose survival was significantly superior to those with other aetiologies, echoing evidence from recently published meta-analyses highlighting improved survival among patients with HCVassociated HCC treated with sorafenib [35].

Regardless of the causality, the diversity in prognostic outlook that we document here is a finding of greater consequence with the advent of second-line therapies for HCC. In our study, PT patients were less likely to receive BSC following sorafenib cessation, making this group an optimally suited patient population for further anticancer treatment upon permanent sorafenib cessation.

Our results may also have important implications for optimizing the sequencing of treatments for patients with HCC. Whilst limited by a non-randomized observational study design, our findings suggest that in patients who are initially eligible to radical/locoregional therapies, these should be prioritized over systemic treatment. The use of sorafenib as "salvage" treatment in the context of disease progression or relapse after radical/locoregional therapies does not seem to negatively affect outcome, being conversely associated with an improved survival probability despite the longer time from the original diagnosis of HCC that characterizes the PT group.

We acknowledge a number of limitations to our observations. Firstly, the multicentre observational nature of this study, whilst limiting systematic bias, is influenced by the heterogeneity in the provision of treatments prior to and after sorafenib therapy, with implications for the estimation of survival. Secondly, we could not adjust our analyses for the type or number of TACE procedures, a treatment strategy characterized by wide interinstitutional variability. Despite the acknowledged limitations, our patient data set is fully representative of the population of patients eligible to sorafenib $[7,8,36]$ : the median OS of 10.4 months in our PT group is similar to that of the patients treated with TACE in the SHARP trial (median OS: 11.9 months) and with TACE or RFA in the Asian-Pacific trial (median OS: 7.3 and 10.5 months, respectively) $[12,13]$. 
To conclude, in this observational study we have shown that TN patients receiving sorafenib as first-line anticancer therapy have profoundly different survival outcomes from those who received it after prior treatment for HCC. We have highlighted the sources of clinical heterogeneity, including stage and liver functional imbalance, underlying this difference. Indirectly, our study supports the provision of sorafenib in the context of treatment migration following failure of radical or locoregional therapies, and highlights that this patient population is optimally suited for second-line therapies. Whilst we could not control for potential confounders - including a different underlying biology, varying burden of disease within each BCLC stage, and a different and heterogeneity in the criteria for conversion from radical/locoregional to sorafenib - taken together, our study suggests that TN and PT patients should be regarded as different clinical entities, a finding that should be carefully weighted in clinical study design as well as in clinical practice.

\section{Acknowledgements}

D.B. is supported by the Berta Ottenstein Programme, Faculty of Medicine, University of Freiburg. D.J.P. is supported by the National Institute for Health Research (NIHR).

\section{Disclosure Statement}

The authors have no conflicts of interest to disclose.

\section{Funding Sources}

No specific funding was obtained to support the conduction of this study.

\section{Author Contributions}

Study concept and design: D.J.P., C.Y.; acquisition of data: D.J.P. and T.A., D.B., H.Y.C., L.R., M.E.B., T.P., M.P., L.G., A.A., M.K., R.T., J.W.P., and R.S.; analysis and interpretation of data: D.J.P., R.S., L.R., and C.Y.; drafting of the manuscript: C.Y. and D.J.P.; critical revision of the manuscript for important intellectual content: all the authors; statistical analysis: C.Y. and D.J.P.; administrative, technical, or material support: R.S., L.R., J.W.P., R.T., M.K., and M.P.; study supervision: D.J.P.

\section{References}

1 Ferlay J, Soerjomataram I, Dikshit R, et al: Cancer incidence and mortality worldwide: sources, methods and major patterns in GLOBOCAN 2012. Int J Cancer 2015;136:E359-E386.

2 Jemal A, Bray F, Center MM, Ferlay J, Ward E, Forman D: Global cancer statistics. CA Cancer J Clin 2011;61: 69-90.

3 American Cancer Society: Global Cancer Facts and Figures, ed 3. Atlanta, American Cancer Society, 2015.

4 Dhir M, Melin AA, Douaiher J, et al: A review and update of treatment options and controversies in the management of hepatocellular carcinoma. Ann Surg 2016;263:1112-1125.

5 Leoni S, Piscaglia F, Serio I, et al: Adherence to AASLD guidelines for the treatment of hepatocellular carcinoma in clinical practice: experience of the Bologna Liver Oncology Group. Dig Liver Dis 2014;46:549-555.

6 Park JW, Chen M, Colombo M, et al: Global patterns of hepatocellular carcinoma management from diagnosis to death: the BRIDGE Study. Liver Int 2015;35:2155-2166.

7 Cheng A-L, Kang Y-K, Chen Z, et al: Efficacy and safety of sorafenib in patients in the Asia-Pacific region with advanced hepatocellular carcinoma: a phase III randomised, double-blind, placebo-controlled trial. Lancet Oncol 2009;10:25-34.

8 Llovet JJM, Ricci S, Mazzaferro V, et al: Sorafenib in advanced hepatocellular carcinoma. N Engl J Med 2008; 359:378-390. 
Yen et al.: Treatment Stage Migration Maximizes Survival Outcomes in Patients with

9 Peck-Radosavljevic M, Greten TF, Lammer J, et al: Consensus on the current use of sorafenib for the treatment of hepatocellular carcinoma. Eur J Gastroenterol Hepatol 2010;22:391-398.

10 European Association for the Study of the Liver; European Organisation for Research and Treatment of Cancer: EASL-EORTC clinical practice guidelines: management of hepatocellular carcinoma. J Hepatol 2012; 56:908-943.

11 Kudo M, Lencioni R, Marrero JA, et al: Regional differences in sorafenib-treated patients with hepatocellular carcinoma: GIDEON observational study. Liver Int 2016;36:1196-1205.

12 Cheng AL, Guan Z, Chen Z, et al: Efficacy and safety of sorafenib in patients with advanced hepatocellular carcinoma according to baseline status: subset analyses of the phase III Sorafenib Asia-Pacific trial. Eur J Cancer 2012;48:1452-1465.

13 Bruix J, Raoul J-L, Sherman M, et al: Efficacy and safety of sorafenib in patients with advanced hepatocellular carcinoma: subanalyses of a phase III trial. J Hepatol 2012;57:821-829.

14 Pressiani T, Boni C, Rimassa L, et al: Sorafenib in patients with Child-Pugh class A and B advanced hepatocellular carcinoma: a prospective feasibility analysis. Ann Oncol 2013;24:406-411.

15 Pinter M, Sieghart W, Graziadei I, et al: Sorafenib in unresectable hepatocellular carcinoma from mild to advanced stage liver cirrhosis. Oncologist 2009;14:70-76.

16 Kudo M: A new era of systemic therapy for hepatocellular carcinoma with regorafenib and lenvatinib. Liver Cancer 2017;6:177-184.

17 El-Khoueiry AB, Sangro B, Yau T, et al: Nivolumab in patients with advanced hepatocellular carcinoma (CheckMate 040): an open-label, non-comparative, phase 1/2 dose escalation and expansion trial. Lancet 2017;389:2492-2502.

18 Rimassa L, Pressiani T, Personeni N, Santoro A: Regorafenib for the treatment of unresectable hepatocellular carcinoma. Expert Rev Anticancer Ther 2017;17:567-576.

19 Johnson PJ, Berhane S, Kagebayashi C, et al: Assessment of liver function in patients with hepatocellular carcinoma: a new evidence-based approach - the ALBI grade. J Clin Oncol 2015;33:550-558.

20 Llovet JM, Brú C, Bruix J: Prognosis of hepatocellular carcinoma: the BCLC staging classification. Semin Liver Dis 1999;19:329-338.

21 Baek KK, Kim J-H, Uhm JE, et al: Prognostic factors in patients with advanced hepatocellular carcinoma treated with sorafenib: a retrospective comparison with previously known prognostic models. Oncology 2011;80: 167-174.

22 Devlin P, Cheng S, Vyas R, Parikh ND: Sorafenib associated survival in treatment naïve versus treatment experienced patients with advanced hepatocellular carcinoma. Gastroenterology 2016;150:S516.

23 Wörns M, Koch S, Niederle IM, et al: The impact of patient and tumour baseline characteristics on the overall survival of patients with advanced hepatocellular carcinoma treated with sorafenib. Dig Liver Dis 2013;45: 408-413.

24 Song T, Zhang W, Wu Q, et al: A single center experience of sorafenib in advanced hepatocellular carcinoma patients. Eur J Gastroenterol Hepatol 2011;23:1233-1238.

25 Bruix J, Takayama T, Mazzaferro V, et al: Adjuvant sorafenib for hepatocellular carcinoma after resection or ablation (STORM): a phase 3, randomised, double-blind, placebo-controlled trial. Lancet Oncol 2015;16: 1344-1354.

26 Lencioni R, Llovet JM, Han G, et al: Sorafenib or placebo plus TACE with doxorubicin-eluting beads for intermediate stage HCC: the SPACE trial. J Hepatol 2016;64:1090-1098.

27 Meyer T, Fox R, Ma YT, et al: TACE 2: a randomized placebo-controlled, double-blinded, phase III trial evaluating sorafenib in combination with transarterial chemoembolisation (TACE) in patients with unresectable hepatocellular carcinoma (HCC) - background. J Clin Oncol 2016;34(suppl):4018.

28 Kudo M, Imanaka K, Chida N, et al: Phase III study of sorafenib after transarterial chemoembolisation in Japanese and Korean patients with unresectable hepatocellular carcinoma. Eur J Cancer 2011;47:2117-2127.

29 A new prognostic system for hepatocellular carcinoma: a retrospective study of 435 patients: the Cancer of the Liver Italian Program (CLIP) investigators. Hepatology 1998;28:751-755.

30 Kudo M, Chung H, Osaki Y: Prognostic staging system for hepatocellular carcinoma (CLIP score): its value and limitations, and a proposal for a new staging system, the Japan Integrated Staging Score (JIS score). J Gastroenterol 2003;38:207-215.

31 Marrero JA, Fontana RJ, Barrat A, et al: Prognosis of hepatocellular carcinoma: comparison of 7 staging systems in an American cohort. Hepatology 2005;41:707-716.

32 Raoul J-L, Bruix J, Greten TF, et al: Relationship between baseline hepatic status and outcome, and effect of sorafenib on liver function: SHARP trial subanalyses. J Hepatol 2012;56:1080-1088.

33 Yau T, Chan P, Ng KK, et al: Phase 2 open-label study of single-agent sorafenib in treating advanced hepatocellular carcinoma in a hepatitis B-endemic Asian population. Cancer 2009;115:428-436.

34 Zhai W, Lim TK-H, Zhang T, et al: The spatial organization of intra-tumour heterogeneity and evolutionary trajectories of metastases in hepatocellular carcinoma. Nat Commun 2017;8:4565.

35 Jackson R, Psarelli E-E, Berhane S, et al: Impact of viral status on survival in patients receiving sorafenib for advanced hepatocellular cancer: a meta-analysis of randomized phase III trials. J Clin Oncol 2017;35:622-628.

36 Johnson PJ, Qin S, Park JW, et al: Brivanib versus sorafenib as first-line therapy in patients with unresectable, advanced hepatocellular carcinoma: results from the randomized phase III BRISK-FL study. J Clin Oncol 2013; $31: 3517-3524$ 